\title{
HIV-1 disease progression is associated with bile- salt stimulated lipase (BSSL) gene polymorphism
}

\author{
Martijn J Stax ${ }^{1 *}$, Neeltje A Kootstra ${ }^{2}$, Angélique B van't Wout², Margreet Bakker', Georgios Pollakis', \\ William A Paxton ${ }^{1}$ \\ From Frontiers of Retrovirology 2011 \\ Amsterdam, The Netherlands. 3-5 October 2011
}

\section{Background}

Among cell types targeted by HIV-1 are dendritic cells (DCs), which play a central role in protection against infections and activation of anti-microbial immune responses. HIV-1 binds pathogen receptor DC-SIGN expressed by $\mathrm{DCs}$ and is transferred to $\mathrm{CD} 4^{+} \mathrm{T}$-lymphocytes. We previously identified BSSL (bile-salt stimulated lipase) as the DC-SIGN binding and HIV-1 transfer blocking glycoprotein in human milk. In vitro DC-SIGN binding properties of human milk associated with a variation in the number of repeats encoded by exon 11 of the BSSL gene. BSSL is not only expressed in milk but also in blood and blood BSSL binds to CXCR4. We hypothesized that BSSL in blood interferes with the interaction of CXCR4 and DC-SIGN with HIV-1 and thus influences HIV-1 infection and HIV-1 disease progression.

\section{Materials and methods}

The relation between BSSL exon 11 repeat numbers and HIV-1 transmission and disease course as well as the emergence of CXCR4-using variants were studied using Kaplan Meier and multivariate Cox proportional hazard analysis. Additionally, the association of BSSL genotype with CD4 cell count was analyzed, both pre-infection and post-infection at viral setpoint. The study group existed of a cohort of men having sex with men and included 334 seropositive and 48 high risk seronegative individuals (HRSN) participating in the Amsterdam Cohort Studies on HIV infection and AIDS.

\section{Results}

The number of repeats in BSSL exon 11 were highly variable ranging from 10 to 18 in seropositive individuals

'Lab of Exp Virology, Academic Medical Center of the Univ of Amsterdam (AMC

Full list of author information is available at the end of the article and from 5-17 in HRSN with 16 repeats being dominant ( $82 \%$ in the seropositive and $85 \%$ in the HRSN group carry at least one allele with 16 repeats). As compared to seropositives, a trend was observed with HRSN having more frequently two alleles with 16 repeats $(\mathrm{p}=0.059)$. We then defined 16 to 18 repeats as high $(\mathrm{H})$ and less than 16 repeats as low (L) repeat numbers. Individuals have either two low (LL), one low and one high (LH) or two high repeat number alleles $(\mathrm{HH})$. The HH BSSL genotype associated with high CD4 cell numbers prior to HIV-1 infection $(\mathrm{p}=0.007)$. No differences were found between BSSL genotypes in CD4 count and virus load at viral setpoint post seroconversion. However, the $\mathrm{HH}$ BSSL genotype was linked to slow disease progression $(\mathrm{p}=0.049, \mathrm{RH}=0.737)$ and delayed emergence of CXCR4using HIV-1 variants $(\mathrm{p}=0.007, \mathrm{RH}=0.515)$.

\section{Conclusion}

We identified BSSL as a marker for HIV-1 disease progression, CXCR4 using HIV-1 variant emergence and CD4 cell homeostasis. Knowledge on the identified BSSL genotypes may help develop a new class of therapeutic molecules aimed at treating HIV-1 disease. (Dutch AIDS fund grant 2005024)

\section{Author details \\ 'Lab of Exp Virology, Academic Medical Center of the Univ of Amsterdam (AMC. 'Dept Exp Immun, Sanquin Res, Landsteiner Lab, and CINIMA, AMC, Amsterdam, The Netherlands.}

Published: 3 October 2011

\section{doi:10.1186/1742-4690-8-S2-P65}

Cite this article as: Stax et al:: HIV-1 disease progression is associated with bile-salt stimulated lipase (BSSL) gene polymorphism. Retrovirology 2011 8(Suppl 2):P65.

\section{Ciomed Central}

(c) 2011 Stax et al; licensee BioMed Central Ltd. This is an open access article distributed under the terms of the Creative Commons Attribution License (http://creativecommons.org/licenses/by/2.0), which permits unrestricted use, distribution, and reproduction in any medium, provided the original work is properly cited. 